\title{
Change in Antiplatelet Therapy in Prevention of Secondary Stroke (CAPS2) Study
}

\author{
Souvik Sen ${ }^{1 *}$, Kolby T Redd ${ }^{1}$, Mihyun J Lim ${ }^{1}$, Ravish Kothari ${ }^{1}$, Julian Duda ${ }^{1}$, Lauren Giamberardino ${ }^{1}$ and William J Powers ${ }^{2}$
}

${ }^{1}$ Department of Neurology, University of South Carolina, Columbia, South Carolina, USA

${ }^{2}$ Department of Neurology, University of North Carolina, Chapel Hill, North Carolina, USA

\begin{abstract}
Goal: Significant proportions of stroke/transient ischemic attack events occur in patients taking daily aspirin. We studied the comparative effectiveness of Food and Drug Administration approved antiplatelet agents (aspirin, clopidogrel, and aspirin-dipyridamole combination) in prevention of recurrent vascular events in stroke/transient ischemic attack patients on aspirin.

Materials and Methods: Consecutive patients having stroke or transient ischemic attack were screened and enrolled into this registry. Patients on long-term dual antiplatelet therapy or oral anticoagulants were excluded. Patient's etiological stroke subtype, medication history, medication compliance, and Aspirin Platelet Function Test were assessed at baseline. Changes in antiplatelet regimen and statin therapy were also recorded. Follow up 6 and 12-month phone visits were conducted to assess primary outcomes of major adverse cardiovascular events that included a composite of death, myocardial infarction and stroke or transient ischemic attack.

Result: One-hundred-eighty subjects (mean age $\pm S D=68 \pm 12$ years, 59\% male, $51 \%$ white, $49 \%$ AfricanAmerican) were enrolled over 24-months. Majority (64\%) had ischemic stroke (NIHSS: $0-21$ ) and remaining (36\%) had transient ischemic attack $\left(A B C D^{2}: 2-7\right)$. Within 12-months of the index cerebrovascular event, $3(5.9 \%)$ participants on clopidogrel, $21(18.8 \%)$ participants on aspirin and $7(41.2 \%)$ participants on aspirin-dipyridamole combination had a composite vascular event (Log rank $p=0.0011)$.

Conclusion: This study shows a significant difference in major adverse cardiovascular event within 12 months of stroke/transient ischemic attack on Aspirin, with effectiveness recorded in the order: clopidogrel>aspirin> aspirindipyridamole combination. A larger randomized pragmatic trial may help ascertain this finding and clarify if specific stroke subtypes benefit from specific antiplatelet agents.
\end{abstract}

Keywords: Aspirin treatment failure; Clopidogrel; Dual antiplatelet therapy; Secondary stroke

\section{Introduction}

Stroke is the leading cause of adult disability, the fifth leading cause of death, and a major source of healthcare cost in the United States [1]. Survivors of a "mini stroke" or transient ischemic attack (TIA) or stroke represent a population at increased risk of subsequent stroke, heart attack, and death [1]. Approximately one quarter of the 795,000 strokes that occur each year are recurrent events [1,2]. Recurrent strokes contribute to the patient's overall disability, suffering, complications and even death in some cases [3]. Patients who present with a first or recurrent stroke are commonly on aspirin for cardiovascular indications $[4,5]$. Despite being on aspirin, they remain at risk of having another stroke, heart attack and/or death [3-5]. Several treatment options are available for prevention of secondary vascular events, including three types of antiplatelet agents, which inhibit platelets that lead to ischemic stroke - higher dose of aspirin $(325 \mathrm{mg})$, clopidogrel, or aspirin with extended release dipyridamole (ASA + DP). These treatment decisions are made without the evidence that the treatments are beneficial and without serious side effects. Lack of current evidence can be attributed to the difficulty and expensive nature of clinical trials. Since we know that each of these antiplatelet agents individually reduces the risk of stroke, heart attack, and death, the use of a placebo would be ethically unacceptable [6-12]. Accordingly, the most recent American Heart Association/American Stroke Association guideline states that there is no evidence that switching or combining antiplatelet agents reduces the risk of subsequent vascular events in patients who have had stroke while receiving aspirin (Class IIb, Level of Evidence C) [11].
A recent meta-analysis of five studies showed that among stroke (TIA) patients on aspirin monotherapy, switching to or addition of another antiplatelet agent reduced the number of major adverse cardiovascular events (MACE) [13]. Four of these studies used clopidogrel, of which two were subgroup analyses of randomized trials namely SPS3 and CHANCE $[14,15]$. A major source of heterogeneity of treatment effect was the SPS3 trial that included lacunar strokes on aspirin and failed to show benefit in the group assigned to clopidogrel [14]. A prospective multicenter stroke registry from South Korea and a retrospective cohort from the Taiwanese national health insurance database have shown that patients experiencing stroke on aspirin had a lower risk of recurrent stroke when switched to clopidogrel compared to maintaining on aspirin [13]. These registries have several deficiencies in terms of applicability of comparative effectiveness data to the US population, including lack of data on medication adherence, data obtained from almost an exclusive Asian population, and lack of information on Aspirin dose at the time of index stroke [13-16]. The Change in Antiplatelet therapy in Prevention of Secondary Stroke (CAPS2 Pilot) registry is a large, simple, relatively low cost,

*Corresponding author: Souvik Sen, Department of Neurology, University of South Carolina, Columbia, South Carolina, USA, Tel: (803) 545-6073 Fax: (803) 545-6066; E-mail: Souvik.Sen@uscmed.sc.edu

Received May 25, 2018; Accepted June 27, 2018; Published June 30, 2018

Citation: Sen S, Redd KT, Lim MJ, Kothari R, Duda J, et al. (2018) Change in Antiplatelet Therapy in Prevention of Secondary Stroke (CAPS2) study. J Neurol Disord 6: 385. doi:10.4172/2329-6895.1000385

Copyright: $\odot 2018$ Sen S, et al. This is an open-access article distributed under the terms of the Creative Commons Attribution License, which permits unrestricted use, distribution, and reproduction in any medium, provided the original author and source are credited. 
observational study that addresses these issues, as well as provides information on comparative effectiveness in the predominantly biracial US population. The registry compared the effect of the three types of antiplatelet agents (aspirin, clopidogrel, or ASA+DP) in prevention of stroke/TIA, heart attack, or death. The data obtained will enable us to design a future large pragmatic randomized clinical trial to further investigate the mentioned comparative effectiveness.

\section{Materials and Methods}

The objective of this research proposal was to compare effectiveness of antiplatelet agents currently being used to prevent recurrent vascular events in stroke/TIA patients already on aspirin at the time of their index stroke/TIA. This was a prospective, longitudinal, observational cohort study using a web-based data capture system and analysis strategies that adjust for confounders. The comparisons evaluated included three types of orally administered antiplatelet agent changes:

1) Aspirin ( $81 \mathrm{mg} \mathrm{mg}$ daily) $\rightarrow$ aspirin ( $325 \mathrm{mg} /$ daily).

2) Aspirin ( $81 \mathrm{mg} / 325 \mathrm{mg}$ daily) $\rightarrow$ clopidogrel (75 mg daily). day).

3) Aspirin $(81 \mathrm{mg} / 325 \mathrm{mg}$ daily $) \rightarrow$ ASA+DP (25-200 mg twice a

The primary study outcome was MACE that included a composite of death, myocardial infarction and stroke/TIA were assessed during the 6 and 12-month follow up phone-calls.

\section{Subjects}

This CAPS2 registry database was established and conducted in 2015 at the Palmetto Health - University of South Carolina School of Medicine Department of Neurology and the Palmetto Health Richland Hospital located in the "stroke belt". The eligibility criteria for this study were:

(1) At least 18 years of age,

(2) TIA $\leq 30$ days with $\mathrm{ABCD}^{2}$ score of $\geq 4$,

(3) Ischemic stroke $\leq 30$ days confirmed by imaging (MRI or CT scan) between January 2015 and July 2017,

(4) Patient on daily antiplatelet therapy with aspirin $(81 / 325 \mathrm{mg})$ for $\geq 30$ days prior to index stroke/TIA,

(5) Prescribed aspirin, clopidogrel, or ASA+DP by his/her physician at discharge, and

(6) Patient or patient's legally appointed representative has been informed of the nature of the study, agrees to participation, and has signed an IRB approved consent form.

We excluded patients with a primary hemorrhagic stroke (intracerebral or subarachnoid hemorrhage), on oral anticoagulation therapy, on clopidogrel or ASA+DP, atrial fibrillation, requiring longterm ( $>21$ days) dual antiplatelet therapy for other indications such as recent acute coronary syndromes or coronary stent placement [17], unable to take medications by mouth, or unable to follow-up for the 12 -month period. The local institutional review board (IRB) approved the study and informed consent was obtained during hospitalization for the index cerebrovascular event. Each subject was assigned a unique study identifier that was confidentially maintained throughout all the data collection processes, including collection of baseline hospitalization, discharge information, and follow-up interviews at 6 and 12 months. This was a prospective cohort study where data was obtained from medical records and clinically validated questionnaires described below. Treatment decision and diagnostic tests were performed at the discretion of the treating physician. Prior to the launch of the registry, a pilot was conducted to test the feasibility of randomization to the three arms: aspirin, clopidogrel and ASA+DP. After random assignment, we engaged the stroke care manager to investigate the patients' health insurance, prescription drug coverage and financial ability to afford the assigned antiplatelet drug. Prior study has shown that engagement of the care manager, the physician and the patient in partnership enhances the medical adherence and improves health outcome in patients with cardiovascular disease [18]. However, this was not sustainable primarily because insurance carriers' coverage did not include ASA+DP, and self-paying patients could not afford to purchase clopidogrel or ASA+DP. It was hence decided to proceed with a registry with non-random assignment guided by physician decision and drug-affordability. This would provide a real-life experience in stroke prevention strategies.

\section{Data collection}

Screening, demographic, clinical, laboratory, and imaging data were collected prospectively through patient report and medical record review. Baseline data, including the National Institutes of Health Stroke Scale (NIHSS), ABCD ${ }^{2}$ score, Trial of ORG 10172 in Acute Stroke Treatment (TOAST) classification for stroke subtype, Modified Rankin Scale (mRS), and Morisky's Medication Adherence Scale were collected on each participant at the time of hospitalization [19-23]. The study subjects were divided into three groups according to the medical regimen they were placed on at discharge; staying on aspirin, changing to clopidogrel, and changing to ASA+ER-DP.

\section{Demographics and risk factors}

Cerebrovascular risk factors were defined as follows: hypertension: previously diagnosed and treated with an antihypertensive medication; diabetes: previously diagnosed with or without treatment with a medication for glycemic control; hypercholesterolemia: previously diagnosed with or without treatment with cholesterol-lowering medications; cigarette smoker: smoking one or more cigarettes daily; alcohol user: alcoholic beverages consumed daily; peripheral vascular disease: symptoms of intermittent claudication or peripheral vascular disease with confirmation by ultrasonographic vascular study or previous peripheral arterial revascularization procedure; ECG and or on ECG monitoring; coronary artery disease, confirmed history of myocardial infarction, angina, or coronary artery revascularization procedure. Data on medications specifically linked to cardiovascular outcomes and used in treatment of hypertension, diabetes and hyperlipidemia were collected. Specific medications included angiotensin converting enzyme inhibitor (ACEI) or angiotensin receptor blocking (ARB) agents, diuretics, used to treat hypertension; oral hypoglycemic agents and insulin used to treat diabetes, moderate and intensive statin therapy used for secondary prevention after stroke/TIA. (17)

\section{Morisky's medication adherence scale}

A validated eight-item Morisky's scale was used to assess selfreported measure of medication-adherence [20,21]. In stroke patients with significant disability, the questionnaire was answered by the next of kin who is also the predominant care giver at home. The theory underlying this measure is that failure to adhere to a medication regimen could occur due to several factors such as "do you sometimes have problems remembering to take your medication", "do you sometimes forget to take your medication," and problems with the complexity of the medical regimen such as, "do you ever feel hassled about sticking to your treatment plan". The questions are phrased to avoid the "yes- 
saying" bias by reversing the wording of the questions regarding the way patients might experience failure in following their medication regimen since there is a tendency for patients to give their physicians or other health care provider's positive answers. Each item is measuring a specific medication-taking behavior and not a determinant of adherence behavior. Response categories are yes/no for each item with a dichotomous response and a 5-point Likert response for the last item. A score of 0-8 was obtained in each subject, with a score 0 indicating high adherence, a score 1 or 2 indicating medium adherence and a score $\geq 2$ indicating low adherence $[20,21]$.

\section{TOAST classification of stroke subtype}

Stroke etiology was classified using the TOAST criteria [22,23]. Briefly, cardioembolic etiology was considered in patients with major brain artery or branch cortical artery occlusion, who had at least one major cardiac source identified in the TOAST criteria. Potential large-artery atherothrombosis was assessed by carotid ultrasound and/or MRA. All tests performed as a part of the initial stroke workup and including transthoracic echocardiography, transesophageal echocardiogram, 12-lead electrocardiogram, and 48-72 hours of cardiac monitoring were used to determine cardiac sources. Small vessel occlusive disease was considered in patients with no evidence of cardioembolism or large artery atherothrombosis described above. These patients should also present with one of the lacunar syndromes and have a corresponding infarct $(<1.5 \mathrm{~cm}$ in its largest diameter) on relevant brainstem or subcortical hemispheric location on CT/MRI.

\section{Laboratory tests}

Fasting lipid profile, high-sensitivity C-reactive protein and aspirin resistance were measured from each patient as part of their admission workup and stroke risk assessments. Serum samples were assayed for fasting lipid profile by the clinical chemistry analyzer Architect c8000 Chemistry System (Abbott, USA); High-sensitivity CRP levels were obtained with a Behring Nephelometer Analyzer and expressed in milligrams per deciliter [24]. Verify Now Aspirin (Accumetrics Inc, San Diego, Calif) is an FDA approved point-of-care device that uses a turbidimetric-based optical detection system to measures plateletinduced aggregation in citrated whole blood. It is used to determine aspirin responsiveness of patients on aspirin. The system measures Aspirin Reaction Units (ARU) as a function of the rate and extent of platelet aggregation. The measured ARU indicate the amount of thromboxane A2-mediated activation of GP IIb/IIIa receptors involved in platelet aggregation. Expected values are in the range of 350-700 ARU. The cut-off to determine if a patient is receiving the therapeutic benefit of aspirin is 549 [24].

\section{Outcomes}

Study data were collected at baseline/discharge, 6 months, and 12 months after the stroke or TIA through medical records review and centralized phone center call surveys. Recurrent vascular events were ascertained and adjudicated after review of medical records. The primary outcome assessment included primary outcomes of MACE that included a composite of vascular death, myocardial infarction, and stroke/TIA. The operational definitions were like the ones used in the Clinical Research Center for Stroke-Fifth Division Registry in South Korea (CRCS-5) registry [25]. Vascular death was defined as death due to stroke, myocardial infarction, or sudden death. This was verified from hospital records and/or death certificate. On the phone, the patient or next of kin were asked if a diagnosis of stroke or TIA was made during hospitalization. Stroke was defined as persistent neurological deficit of sudden onset (non-convulsive) lasting 24 hours or longer with CT/MRI confirmation. TIA was defined as sudden onset neurological deficit that resolved within 24 hours with no CT/MRI evidence of new cerebral infarct. Patients suspected of complicated migraine or seizures or those presenting with vague neurological symptoms were excluded. On the phone the patient/next of kin were asked if a diagnosis of "heart attack" was made during hospitalization. Myocardial infarction was defined as clinical features, including typical chest pain, electrocardiographic (EKG) changes and elevated levels of troponins verified on medical records. The data was entered into web-based case report forms in StudyTrax. StudyTrax is a clinical trial management system with full HIPAA compliance and utilizes a web-based portal access.

\section{Statistical analysis}

Demographics including age, sex, and race as well as the investigated additional covariates including socioeconomic status, hypertension, diabetes, hypercholesterolemia, carotid stenosis, coronary artery disease, obstructive sleep apnea, peripheral vascular disease, body mass index (BMI), smoking status, drug use, current alcohol use, stroke etiological classification, fasting lipid profile, and High sensitivity C-reactive protein (HS-CRP) levels were assessed as a part of baseline characteristics. Crude and adjusted hazard ratio (HR) and 95\% confidence intervals were all calculated using Cox proportional hazards models to analyze the association among subjects on Clopidogrel and ASA+DP with a recurrent event in comparison with those on aspirin. Adjusted HR analysis was performed by adjusting for covariates including age, sex, race, hypertension, diabetes, hypercholesterolemia, carotid stenosis, coronary artery disease, obstructive sleep apnea, peripheral vascular disease, smoking status, and medications specifically linked to cardiovascular outcome. A covariate was considered to be a confounder if $\mathrm{a} \geq 10 \%$ difference was noted between unadjusted HR and HR adjusted for the covariate and included in the final Cox proportional hazard model to obtain the adjusted HR for the specific antiplatelet drug (clopidogrel or ASA+DP). Further evaluation of patients on the three antiplatelet drug treatment groups was carried out by generating a Kaplan Meier survival from recurrent vascular event curves and testing for differences using the log-rank test. All data analysis for this study was completed using SAS version 9.4 (SAS institute Inc., Cary, NC).

\section{Results}

One-hundred-eighty-two subjects were enrolled over a 24-month period, of which 2 were switched to oral anticoagulation after detection of new onset atrial fibrillation and excluded from our data analysis. The remaining 180 (mean age $\pm S D=68 \pm 12$ years, $59 \%$ males, $51 \%$ white, $49 \%$ African-American) were followed for a period of 12 months. Majority (64\%) had ischemic stroke (NIHSS: 0-21) and remaining (36\%) had TIA $\left(\mathrm{ABCD}^{2}: 2-7\right)$. Ischemic strokes were attributed to large artery atherothrombosis $(41 \%)$, small vessel occlusive disease $(7 \%)$, undetermined (16\%), cardioembolism (34\%) and others (2\%). Majority of the patients were on $81 \mathrm{mg}$ of aspirin (75\%) and remainder on $325 \mathrm{mg}$ of aspirin (25\%). Of the 180 subjects, 112 were continued on aspirin (81-325 mg/ day), 51 prescribed clopidogrel, and the remaining 17 were prescribed ASA+DP. The baseline clinical characteristics of these subjects are shown in (Table 1). There were no significant differences among these three groups in demographics, education, annual household income, vascular risk factors, social risk factors, and etiological stroke subtypes. Using the Morisky Adherence scale, none of the subjects had high medication adherence. A third to half of the subjects demonstrated low medication adherence and the remaining subjects demonstrated medium medication adherence. The three groups demonstrated similar levels of aspirin platelet function measured as ARU, fasting lipid 
Citation: Sen S, Redd KT, Lim MJ, Kothari R, Duda J, et al. (2018) Change in Antiplatelet Therapy in Prevention of Secondary Stroke (CAPS2) study. J Neurol Disord 6: 385. doi:10.4172/2329-6895.1000385

Page 4 of 7

\begin{tabular}{|c|c|c|c|}
\hline Characteristic & Aspirin $(\mathrm{N}=112)$ & Clopidogrel $(\mathrm{N}=51)$ & $A S A+D P(N=17)$ \\
\hline Age & $68.7 \pm 11.9$ & $66.6 \pm 11.7$ & $67.7 \pm 11.5$ \\
\hline \multicolumn{4}{|l|}{ Sex } \\
\hline Male & $64(57 \%)$ & $36(71 \%)$ & $7(41 \%)$ \\
\hline Female & $48(43 \%)$ & $15(29 \%)$ & $10(59 \%)$ \\
\hline \multicolumn{4}{|l|}{ Race } \\
\hline white & $55(49 \%)$ & $27(53 \%)$ & $10(59 \%)$ \\
\hline African-American & $57(51 \%)$ & $24(47 \%)$ & $7(41 \%)$ \\
\hline \multicolumn{4}{|l|}{ Education } \\
\hline Basic ( $\leq 8$ years) & $7(6 \%)$ & $3(6 \%)$ & $0(0 \%)$ \\
\hline Intermediate ( $\leq 12$ years $)$ & $44(40 \%)$ & $14(29 \%)$ & $4(24 \%)$ \\
\hline Advanced (>12 years) & $49(44 \%)$ & $27(56 \%)$ & $12(71 \%)$ \\
\hline Refused/Unknown & $11(10 \%)$ & $4(25 \%)$ & $1(6 \%)$ \\
\hline \multicolumn{4}{|l|}{ Annual household Income } \\
\hline$<45,000$ & $47(42 \%)$ & $18(38 \%)$ & $8(50 \%)$ \\
\hline $45,000-49,999$ & $3(4 \%)$ & $2(4 \%)$ & $1(6 \%)$ \\
\hline $50,000-59,999$ & $7(6 \%)$ & $4(8 \%)$ & $1(6 \%)$ \\
\hline$\geq 60,000$ & $27(24 \%)$ & $14(29 \%)$ & $2(13 \%)$ \\
\hline Refused/Unknown & $27(24 \%)$ & $10(21 \%)$ & $4(25 \%)$ \\
\hline \multicolumn{4}{|l|}{ Risk factors } \\
\hline Hypertension & $103(92 \%)$ & $45(88.24 \%)$ & $16(94 \%)$ \\
\hline Diabetes & $55(49 \%)$ & $24(47.06 \%)$ & $9(53 \%)$ \\
\hline Hypercholesterolemia & $111(99 \%)$ & $51(100 \%)$ & $16(94 \%)$ \\
\hline Carotid stenosis & $33(30 \%)$ & $16(31 \%)$ & $4(24 \%)$ \\
\hline Coronary artery disease & $43(38 \%)$ & $21(41 \%)$ & $3(18 \%)$ \\
\hline Obstructive sleep apnea & $24(21 \%)$ & $11(22 \%)$ & $1(6 \%)$ \\
\hline Peripheral vascular disease & $5(5 \%)$ & $1(2 \%)$ & $1(6 \%)$ \\
\hline Prior Stroke/TIA & $48(43 \%)$ & $23(45 \%)$ & $11(65 \%)$ \\
\hline Smoking & $54(48 \%)$ & $30(59 \%)$ & $8(47 \%)$ \\
\hline Illicit drug & $1(1 \%)$ & $1(2 \%)$ & $1(6 \%)$ \\
\hline Alcohol use & $45(40 \%)$ & $19(38 \%)$ & $6(35 \%)$ \\
\hline BMI & $31.5 \pm 10.9$ & $32.1 \pm 9.5$ & $28.9 \pm 5.6$ \\
\hline \multicolumn{4}{|l|}{ TOAST Classification } \\
\hline Large-artery atherosclerosis & $44(39 \%)$ & $14(28 \%)$ & $6(35 \%)$ \\
\hline Cardioembolism & $24(21 \%)$ & $11(22 \%)$ & $4(24 \%)$ \\
\hline Small-vessel occlusion & $5(5 \%)$ & $3(6 \%)$ & $0(0 \%)$ \\
\hline Undetermined Etiology & $29(26 \%)$ & $16(31 \%)$ & $3(18 \%)$ \\
\hline Other determined etiology & $0(0 \%)$ & $2(4 \%)$ & $0(0 \%)$ \\
\hline TIA & $10(9 \%)$ & $5(10 \%)$ & $4(23.53 \%)$ \\
\hline \multicolumn{4}{|l|}{ Aspirin dose prior to index event } \\
\hline $81 \mathrm{mg} . \mathrm{PO}$ daily & $87(78 \%)$ & $35(69 \%)$ & $13(76.47 \%)$ \\
\hline 325 mg. PO daily & $25(22 \%)$ & $16(31 \%)$ & $4(23.53 \%)$ \\
\hline \multicolumn{4}{|l|}{ Statin therapy at discharge } \\
\hline Any & $102(91 \%)$ & $49(96 \%)$ & $14(82 \%)$ \\
\hline Intensive & $38(34 \%)$ & $22(43 \%)$ & $6(35 \%)$ \\
\hline \multicolumn{4}{|l|}{ Diabetes medications at discharge } \\
\hline Oral hypoglycemic & $33(30 \%)$ & $20(39 \%)$ & $4(24 \%)$ \\
\hline Insulin & $20(18 \%)$ & $10(20 \%)$ & $3(18 \%)$ \\
\hline \multicolumn{4}{|l|}{ Blood pressure medications at discharge } \\
\hline Any & $98(88 \%)$ & $46(90 \%)$ & $15(88 \%)$ \\
\hline ACE inhibitor/ARB & $66(59 \%)$ & $31(61 \%)$ & $14(82 \%)$ \\
\hline Diuretic & $40(36 \%)$ & $15(48 \%)$ & $8(47 \%)$ \\
\hline \multicolumn{4}{|l|}{ Morisky Adherence Scale } \\
\hline 0 & $0(0 \%)$ & $0(0 \%)$ & $0(0 \%)$ \\
\hline $1-2$ & $60(56 \%)$ & $32(67 \%)$ & $7(44 \%)$ \\
\hline $3-8$ & $47(44 \%)$ & $16(33 \%)$ & $9(56 \%)$ \\
\hline
\end{tabular}

Table 1: Baseline characteristics and risk factors of subjects who had a stroke while on aspirin and then increased aspirin dose, changed to clopidogrel, or to aspirin with dipyridamole (ASA + DP).

profile and high sensitivity C-reactive protein (Table 2). Of the 180 patients, all (100\%) completed the 6-month phone visit and 172 (96\%) completed the 12-month phone-visit. Within 12-months of the index cerebrovascular event (stroke or TIA), 3 (5.9\% 3 stroke, 0 MI, 0 CV deaths) of the participants on clopidogrel, 21 (18.8\%, 13 strokes, $2 \mathrm{MI}$, $6 \mathrm{CV}$ death) of the participants on aspirin and 7 (41.2\% 5 stroke, $2 \mathrm{MI}$, 
Citation: Sen S, Redd KT, Lim MJ, Kothari R, Duda J, et al. (2018) Change in Antiplatelet Therapy in Prevention of Secondary Stroke (CAPS2) study. J Neurol Disord 6: 385. doi:10.4172/2329-6895.1000385

Page 5 of 7

\begin{tabular}{|c|c|c|c|}
\hline Characteristic & Aspirin $(\mathrm{N}=112)$ & Clopidogrel $(\mathrm{N}=51)$ & $A S A+D P(N=17)$ \\
\hline \multicolumn{4}{|l|}{ Aspirin platelet function test (ARU) } \\
\hline Normal $(350-549)$ & $54(78 \%)$ & $30(73 \%)$ & $9(90 \%)$ \\
\hline Abnormal $(550+)$ & $15(22 \%)$ & $11(27 \%)$ & $1(10 \%)$ \\
\hline \multicolumn{4}{|l|}{ Fasting lipid profile } \\
\hline Total Cholesterol (mg/dL) & $168.8 \pm 47.9$ & $157.98 \pm 33.63$ & $158.25 \pm 443.71$ \\
\hline LDL Cholesterol (mg/dL) & $97.1 \pm 40.7$ & $886.29 \pm 29.97$ & $89.25 \pm 41.73$ \\
\hline HDL Cholesterol (mg/dL) & $48.7 \pm 15.2$ & $48.38 \pm 13.96$ & $42.94 \pm 11.65$ \\
\hline Triglycerides (mg/dL) & $129.0(92.0-178.5)$ & $118.0(87.5-179.0)$ & $124.50(99.5-271.5)$ \\
\hline HS-CRP (mg/dL) & $4.0(1.7-9.5)$ & $2.15(0.9-4.5)$ & $3.9(1.3-13.3)$ \\
\hline
\end{tabular}

Table 2: Selected laboratory characteristics.

\begin{tabular}{|c|c|c|c|c|}
\hline Characteristic & Clopidogrel & Change (\%) & ASA+DP & Change (\%) \\
\hline Unadjusted & 0.29 & -- & 2.83 & -- \\
\hline Age & 0.30 & 3.4 & 2.76 & -2.5 \\
\hline Sex & 0.28 & -3.4 & 2.82 & -0.4 \\
\hline Race & 0.29 & 0.0 & 2.67 & -5.7 \\
\hline Hypertension & 0.29 & 0.0 & 2.76 & -2.5 \\
\hline Diabetes & 0.30 & 3.4 & 2.73 & -3.5 \\
\hline Hypercholesterolemia & 0.29 & 0.0 & 2.97 & 4.9 \\
\hline Carotid stenosis & 0.29 & 0.0 & 2.72 & -3.9 \\
\hline Coronary artery disease & 0.29 & 0.0 & 3.02 & 6.7 \\
\hline Obstructive sleep apnea & 0.29 & 0.0 & 2.84 & 0.4 \\
\hline Peripheral vascular disease & 0.29 & 0.0 & 2.85 & 0.7 \\
\hline Smoking & 0.32 & 10.3 & 2.92 & 3.2 \\
\hline Statin therapy (any) & 0.30 & 3.4 & 2.62 & -7.4 \\
\hline Statin therapy (intensive) & 0.28 & -3.4 & 2.76 & -2.5 \\
\hline BP medication (any) & 0.30 & 3.4 & 2.76 & -2.5 \\
\hline BP medication (ACEI/ARB) & 0.30 & 3.4 & 3.13 & 10.6 \\
\hline BP medication (diuretic) & 0.29 & 0.0 & 2.81 & -0.7 \\
\hline Oral hypoglycemics & 0.30 & 3.4 & 2.75 & -2.8 \\
\hline Insulin & 0.29 & 0.0 & 2.80 & -1.1 \\
\hline
\end{tabular}

Table 3: Assessment for confounding: Hazard ratios for the association of antiplatelet agent (aspirin, clopidogrel and aspirin with extended release dipyridamole) and recurrent vascular event (stroke, MI and cardiovascular death) adjusted for individual covariate assessed for confounding.

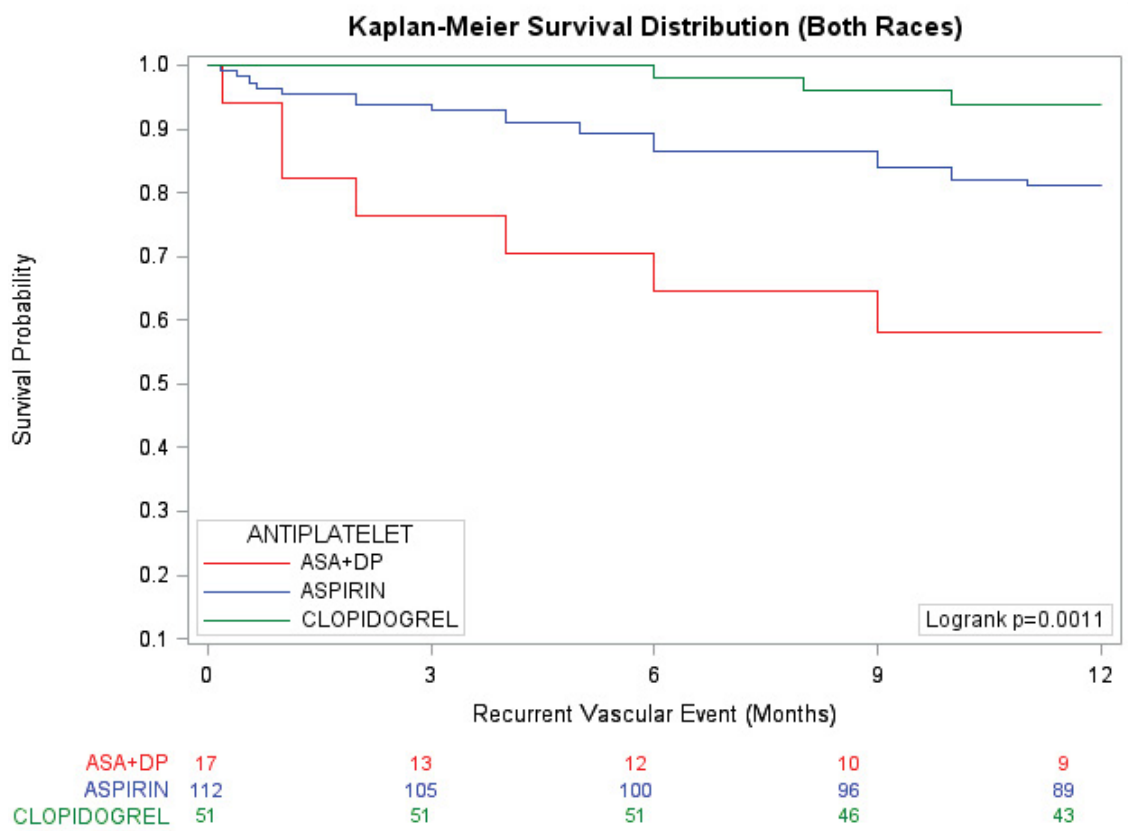

Figure 1: Kaplan Meier curves depicting 12-month outcome of recurrent vascular events in subjects on Aspirin (blue), Clopidogrel (green) and those on aspirin with extended release dipyridamole or ASA+ER-DP (red). Inset: Log rank $p$ value. 


\begin{tabular}{|c|c|c|c|c|c|c|}
\hline Antiplatelet agent & Crude hazard ratio & $95 \% \mathrm{Cl}$ & $\mathbf{p}$ & $\begin{array}{c}\text { Adjusted* } \\
\text { hazard ratio }\end{array}$ & $95 \% \mathrm{Cl}$ & $\mathbf{p}$ \\
\hline aspirin $(\mathrm{N}=112)$ & 1.0 & Ref. & -- & 1.0 & Ref. & -- \\
\hline clopidogrel $(\mathrm{N}=51)$ & 0.29 & $0.09-0.99$ & 0.047 & 0.32 & $0.10-0.99$ & 0.047 \\
\hline$A S A+D P(N=17)$ & 2.83 & $1.20-6.68$ & 0.018 & 3.13 & $1.31-7.49$ & 0.011 \\
\hline
\end{tabular}

Table 4: Hazard ratios (95\% confidence intervals [Cl]) for the association of antiplatelet agent (aspirin, clopidogrel and aspirin with extended release dipyridamole) and recurrent vascular event (stroke, $\mathrm{Ml}$ and cardiovascular death).

$0 \mathrm{CV}$ death) of the participants on ASA+DP had a primary outcome of combined vascular event (Log rank $\mathrm{p}=0.0011)$, depicted in the KaplanMeier curve (Figure 1). The factors tested for confounding included age, sex, race, vascular risk factors and medications (Table 3). Among these covariates only smoking status influenced $(\geq 10 \%$ difference between unadjusted HR and HR adjusted for smoking status) the association between clopidogrel and secondary vascular outcome. ACEI/ARB influenced $(\geq 10 \%$ difference between unadjusted $\mathrm{HR}$ and $\mathrm{HR}$ adjusted for ACEI/ARB) the association between ASA+DP and secondary vascular outcome. Therefore, the unadjusted HR and the HR adjusted for these specific covariates are reported in (Table 4). Compared to the reference group that was continued on aspirin, the clopidogrel group had a significantly lower rate of recurrent vascular event (HR 0.29, 95\% CI 0.09-0.99) and the ASA+DP group had a significantly higher rate of recurrent vascular event (HR 2.83, 95\% CI 1.20-6.68) as depicted in (Table 4). The significance of these associations remained unchanged after the specific adjustments.

The effect of race was tested by introducing a race-antiplatelet therapy interaction term in the hazards ratio model, as well as stratified analysis. The interaction term hazards ratio remained non-significant ( $p=0.34$ ), whereas stratified analyses which may be underpowered, but showed some differences. In African American subjects, compared to the reference group that was continued on aspirin, the clopidogrel group did not reveal a significant difference in recurrent vascular event (HR 0.52, 95\% CA 0.11-2.40) and the ASA+DP group had significantly higher rate of recurrent vascular event (HR 3.59, 95\% CI 0.95-13.58) that achieved borderline significance $(\mathrm{p}=0.06)$. In the White subjects, compared to the reference group that was continued on aspirin, the clopidogrel group had fewer recurrent vascular events (HR 0.14, 95\% CI $0.02-1.12)$ that reached borderline significance $(p=0.06)$ and the ASA+DP group had a non-significant higher rate of recurrent vascular event (HR 2.42, 95\% CI 0.76-7.63, $\mathrm{p}=0.13$ ).

Of note, most subjects were on $81 \mathrm{mg}$ of aspirin $(\mathrm{N}=135,75 \%)$ prior to the index cerebrovascular event. Of these 90 subjects were retained on aspirin (66 at a higher dose of $325 \mathrm{mg}$ and 24 on the same dose of $81 \mathrm{mg}$ ). Among the 24 subjects who were retained on the same dose of aspirin $(81 \mathrm{mg}) 5 \mathrm{had}$ recurrent vascular events and among the 66 of who the dose was increased, 11 had recurrent vascular events (Log rank $\mathrm{p}=0.58$ ). Thus, a change in aspirin dose from $81 \mathrm{mg}$ to $325 \mathrm{mg}$ did not appear to impact the rate of recurrent vascular event. Also, most of the subjects $(139,77 \%)$ were on some form of statin. At the time of discharge $165(85 \%)$ subjects were discharged on a statin. In 75 subjects, statin dose or medication was changed of whom 15 had recurrent vascular event. In 90 subjects the statin dose or the medication remained unchanged, of whom 14 had recurrent vascular events. Thus, change in statin dose or medication did not impact the rates of recurrent vascular event (Log rank p value 0.34).

\section{Discussion}

This single center, prospective, longitudinal registry shows a significant difference in secondary vascular events within 12 months of stroke/TIA on aspirin, with effectiveness recorded in the following order: clopidogrel $>$ aspirin $>$ ASA+DP. The results are consistent with those reported by the registry out of South Korea and the Taiwanese database, in that it showed that subjects on clopidogrel had a lower rate of recurrent vascular events compared to a reference group that was maintained on aspirin [13-15]. Despite fewer numbers of subjects that were placed on ASA+DP, this group appears to have a higher rate of recurrent vascular events. Subgroup analysis of the SPS3 randomized clinical trial, failed to show a difference between aspirin and clopidogrel in prevention of recurrent vascular event in a cohort of lacunar stroke patients on aspirin [14]. A meta-analysis, combining the three above-mentioned study, with two other trials, showed a combined benefit in prevention of MACE and recurrent stroke, by changing antiplatelet therapy agent, compared with maintenance of aspirin [13]. These findings need to be tested in a larger randomized pragmatic trial to ascertain these findings that may impact the clinical practice of secondary prevention strategy in patients experiencing a cerebrovascular event on aspirin.

In addition to the above findings, we report a few others that are relatively unique to this registry. First, we report high rates of medium to high levels of medicine non-adherence in this cohort of patients experiencing a cerebrovascular event on aspirin. Since aspirin is largely a non-prescription medication, it is possible that the aspirin group experiences a high rate of recurrent vascular events stemming from non-adherence to aspirin. Future randomized trials may need to address the issue whether clopidogrel is truly more effective compared to an aspirin adherent group. Non-adherence to aspirin may serve as an explanation of the benefit noted in the observational study and not in the randomized clinical trial SPS3, where aspirin and clopidogrel were provided for as prescription study medication [14]. Strategies to improve medical adherence to aspirin and other cardiovascular medication such as an electronic medication reminder, motivational interviews, and aspirin use as a prescribed medication may be considered.

Based on the results from this registry, we did not find a significant racial disparity in effectiveness of antiplatelet agents in prevention of secondary vascular events. In the overall biracial population, it appears that clopidogrel is significantly more effective than aspirin, whereas $\mathrm{ASA}+\mathrm{DP}$ is significantly less effective than aspirin in prevention of recurrent vascular events. Stratified analysis based on the races, appeared to be underpowered to test racial disparity in effectiveness of antiplatelet agents between African-American and white patients. Clopidogrel is not significantly more effective than aspirin, whereas ASA+DP showed a trend towards less effectiveness in secondary prevention than aspirin in prevention of recurrent vascular events $(\mathrm{p}=0.06)$. In the white race, clopidogrel showed a trend towards more effectiveness than aspirin $(p=0.06)$, whereas ASA+DP is not significantly less effective than aspirin in prevention of recurrent vascular events. Whether the race is truly an effect modifier of the effect of antiplatelet therapy in recurrent vascular events will need to be tested in an adequately powered larger randomized clinical trial.

Additionally, we report the lack of effectiveness of change in dose of aspirin from 81 to $325 \mathrm{mg} /$ day on the rate of recurrent vascular 
Citation: Sen S, Redd KT, Lim MJ, Kothari R, Duda J, et al. (2018) Change in Antiplatelet Therapy in Prevention of Secondary Stroke (CAPS2) study. J Neurol Disord 6: 385. doi:10.4172/2329-6895.1000385

Page 7 of 7

event. Prior studies have shown that higher doses of aspirin translate to lower rates of aspirin resistance [26]. However, these results have not translated to a higher dose leading to a higher effectiveness in prevention of vascular events [5-10]. Our findings are consistent with these results. The current guidelines recommend the use of maximal dose of statin and specific statins-Atorvastatin $80 \mathrm{mg} /$ day or Rosuvastatin $20 \mathrm{mg} /$ day for secondary prevention [11]. However, the results from this registry suggest that change in dose of statin or switching to these specific statins does not significantly reduce the rate of recurrent vascular event. These findings will also need to be verified in a larger randomized clinical trial with longer duration follow-up.

The single-center registry has few weaknesses. First, antiplatelet therapy and statin therapy were assigned based on the preference of the treating physician and hence not randomized. Due to reasons of insurance coverage there is an imbalance in the group size that may impact the results of the study. Despite these weaknesses, the study provides some important information that may help in the designing of a future large randomized pragmatic trial and may help the treating physician make some important clinical decisions regarding antiplatelet therapy and statin therapy in patients encountering cerebrovascular event on aspirin.

Consistent with other registries we report a significantly higher effectiveness of clopidogrel compared to aspirin in the prevention of recurrent vascular event [13]. However, it is known that clopidogrel is a prodrug that relies on enzymatic conversion to activate the drug (cytochrome 450 (CYP)), which is influenced by genetic polymorphism [27]. It may be of value to note if there are racial disparities in these genetic polymorphisms.

\section{Conclusion}

We report a significant difference in secondary vascular eventS within 12 months of stroke/TIA on aspirin, with effectiveness recorded in the order: clopidogrel $>$ aspirin $>A S A+D P$. Based on this finding and findings in other registries, treating physicians may consider switching patients to clopidogrel. We report a high rate of medium to high levels of medical non-adherence in subjects encountering cerebrovascular events on aspirin, a possible effect modification by race and lack of obvious benefits from changing dose of aspirin and/or changing dose and type of statins. These findings need to be verified in large randomized pragmatic trial.

\section{References}

1. CDC (2005) Prevalence and most common causes of disability among adults CDC, USA.

2. Brown DL, Lisabeth LD, Roychoudhury C, Ye Y, Morgenstern LB (2005) Recurrent stroke risk is higher than cardiac event risk after initial stroke/ transient ischemic attack. Stroke 36: 1285-1287.

3. Stafford RS (2000) Aspirin use is low among united states outpatients with coronary artery disease. Circulation 101: 1097-1101.

4. Bayer (2011) Facts about aspirin. Bayer Pharmaceuticals, Germany.

5. Weisman SM, Graham DY (2002) Evaluation of the benefits and risks of lowdose aspirin in the secondary prevention of cardiovascular and cerebrovascular events. Arch Intern Med 162: 2197-2202.

6. Gent M, Beaumont D, Blanchard J, Bousser MG, Coffman J et al. (1996) A randomized, blinded, trial of clopidogrel versus aspirin in patients at risk of ischemic events (CAPRIE). Lancet 348: 1329-1339.

7. Collins R, Peto R, Baigent C, Sandercock EP, Dunbabin D, et al. (1994) Collaborative overview of randomized trials of antiplatelet therapy prevention of death, myocardial infarction, and stroke by prolonged antiplatelet therapy in various categories of patients. BMJ 308: 81-106.

8. Farrell B, Godwin J, Richards S, Warlow C (1991) The United Kingdom transient ischemic attack (UK-TIA) aspirin trial: Final results. J Neurol Neurosurg Psychiatry 54: 1044-4054.

9. He J, Whelton P, Vu B, Klaget MJ (1998) Aspirin and risk of hemorrhagic stroke: A meta-analysis of randomized controlled trials. JAMA 280: 1930-1935.

10. Baigent C, Sudlow C, Collins R (2002) Collaborative meta-analysis of randomized trials of antiplatelet therapy for prevention of death, myocardial infarction, and stroke in high risk patients. BMJ 324: 71-86.

11. Powers WJ, Rabinstein AA, Ackerson T, Adevoe OM, Bambakidis NC, et al. (2018) Guidelines for early management of patients with acute ischemic stroke A guideline for healthcare professionals from the American Heart Association/ American Stroke Association. Stroke 49: e46-e110.

12. Kim JT, Park MS, Choi KH, Cho KH, Kim BJ, et al. (2016) Different antiplatele strategies in patients with new ischemic stroke while taking aspirin. Stroke 47 128-134.

13. Lee M, Saver J, Ovbiagele B (2017) Response by Lee et al to letter regarding article, Pioglitazone for secondary stroke prevention: A systematic review and meta-analysis. Stoke 48: e136-e136.

14. Cote R, Zhang Y, Hart RG, Mc Clure LA, Anderson DC, et al. (2014) ASA failure : Does the combination ASA/clopidogrel confer between long term vascular protection ? Neurology 82: 382-389.

15. Wang Y, Wang Y, Zhao X, Liu L, Wang D, et al. (2013) Clopidogrel with aspirin in acute minor stroke or transient ischemic attack. NEJM 369: 11-19.

16. Wang Y, Zhao X, Lin J, Li H, Johnston SC, et al. (2016) Association between CYP2C19 loss-of-function allele status and efficacy of clopidogrel for risk reduction among patients with minor stroke or transient ischemic attack. JAMA 316: 70-78.

17. Kernan WN, Obviagele B, Black HR, Bravata DM, Chimowitz MI, et al. (2014) Guidelines for the prevention of stroke in patients with stroke and transient ischemic attack: A guideline for healthcare professionals from the American Heart Association/American Stroke Association. Stroke 45: 2160-236.

18. Ciccone MM, Aquilino A, Cortese F, Scicchitano P, Sassara M, et al. (2010) Feasibility and effectiveness of a disease and care management model in the primary health care system for patients with heart failure and diabetes (Project Leonardo). Vasc Health Risk Manag. 6: 297-305

19. Kasner SE (2006) Clinical interpretation and use of stroke scales. Lancet 5 603-612.

20. Morisky DE, Ang A, Krousel-Wood M, Ward HJ (2008) Predictive validity of a medication adherence measure in an outpatient setting. J Clin Hypertens 10: 348-354.

21. Morisky DE, Green LW, Levine DM (1986) Concurrent and predictive validity of a self-reported measure of medication adherence. Med Care 24: 67-74.

22. Chung JW, Park SH, Kim N, Kim WJ, Park JH, et al. (2014) Trial of ORG 10172 in acute stroke treatment (TOAST) classification and vascular territory of ischemic stroke lesions diagnosed by diffusion-weighted imaging. J Am Heart Assoc 3: 1-9.

23. Adams HP, Bendixen BH, Kappelle LJ, Biller J, Love BB, et al. (1993) Classification of subtype of acute ischemic stroke - definitions for use in a multicenter clinical trial. Stroke 24: 35-41.

24. Fink PC, Romer M, Haeckel R (1989) Measurement of proteins with the Behring Nephelometer: A multicentral evaluation. J Clin Chem Clin 27: 261-276.

25. Kim BJ, Park JM, Kang K, Lee SJ, Ko Y, et al. (2015) ERRATUM: Table correction: Case characteristics, hyperacute treatment, and outcome information from the clinical research center for stroke-fifth division registry in South Korea. J Stroke 17: 377-378.

26. Lee PY, Chen WH, Ng W, Cheng X, Kwok, JYY, et al. (2005) Low-dose aspirin increases aspirin resistance in patients with coronary artery disease. Am J Med 118:723-727.

27. Plavix (2011) Diminished antiplatelet effect in patients with two loss-of-function alleles of the Cyp2c19 gene (clopidogrel bisulfate). 